\title{
Dual Toll Pricing for Hazardous Materials Transport with Linear Delay
}

\author{
Jiashan Wang Yingying Kang Changhyun Kwon* Rajan Batta \\ Department of Industrial and Systems Engineering \\ University at Buffalo, SUNY
}

\begin{abstract}
In this paper, we propose a dual toll pricing method to mitigate risk of hazardous materials (hazmat) transportation. We aim to simultaneously control both regular and hazmat vehicles to reduce the risk. In our model, we incorporate a new risk measure to consider durationpopulation-frequency of hazmat exposure. We first formulate the model as a Mathematical Program with Equilibrium Constraints (MPEC). Then we decompose the MPEC formulation into first-stage and second-stage problems. Separate methods are developed to solve each stage. A numerical example is provided and possible extensions are discussed.
\end{abstract}

Keywords: hazardous materials; toll pricing; congestion; risk

\section{Introduction}

Hazardous materials (hazmat) transportation, traffic congestion and traffic safety are the three main transportation issues cited by the National Conference of State Legislatures (NCSL). Traffic safety is an important public health issue for many people, including state legislators. Each year, more than 40,000 people are killed in motor vehicle crashes and thousands more are injured (Movassaghi et al., 2007). Traffic crashes have been found to be a leading cause of death for all age groups in the United States and cost our society an estimated $\$ 230.6$ billion each year.

Traffic congestion and hazmat transportation are generally recognized as two main causes of many traffic safety issues. Traffic congestion does not only delay travel time, but also is the trigger of vehicles crashes and often enlarges the consequences of traffic accidents. But the most costly consequences come from hazmat transportation. Hazmat accidents are well known as lowprobability, high-consequence (LPHC) incidents. On one hand, the number of accidents caused by hazmat shipments are rather low. In USA, in 1998, there were roughly 15,000 accidents related

${ }^{*}$ Corresponding Author. E-mail:chkwon@buffalo.edu 
to hazmat transportation, and only 429 of them were classified as serious incidents (Kara and Verter, 2004). On the other hand, hazmat accidents can result in a large amount of damage to the population and the environment. In 2003, the U.S. Department of Transportation received 14,660 reports of incidents from carriers and shippers, with about 87 percent involving trucks, with a total of $\$ 338,136,186$ in damages (Gallamore et al., 2005). Gasoline was by far the most common material involved, as might be expected given the prevalence of tank trucks on the highways. Together, gasoline, other flammable liquids, and corrosives account for 57 percent of serious incidents. Even though most reported incidents do not involve serious consequences, the annual statistics reveal some highly costly consequences. For instance, the 1996 figures include the chlorine gas escaping from a tank car damaged in a train derailment in Alberton, Montana, resulted in the evacuation of more than 1,000 people, more than 700 injuries, and 1 fatality. The 1998 statistics include the deaths of five people from gasoline that spilled and ignited during the unloading of a tank truck in Biloxi, Mississippi. The incident also resulted in the evacuation of 80 people and the closing of an Interstate highway. Because such major incidents are rare, they stand out and tend to dominate the safety data when they do occur.

Due to the inherent transport risks, municipal units usually try to separate the hazmat flow from normal traffic flow, especially high-density traffic flows. Currently the transportation of hazmat is regulated under the Federal Hazardous Materials Transportation Act (which was amended by the Patriot Act in 2001) in the United States and under the Federal Transportation of Dangerous Goods Act in Canada. In North America and Europe, government agencies do not have the authority to dictate routes to hazmat carriers for moving their shipments. These agencies mitigate the hazmat transport risks by imposing (permanent or time-based) curfews on the use of the road segments under their jurisdiction. An example of this is the ban on trucks carrying non-radioactive hazmat on certain road segments (e.g., Texas Department of Transportation, 2009, has a list of prohibited roads for Texas). These kinds of policies are usually categorized as network-design (ND) policies and much work has been done in this field (e.g., Kara and Verter, 2004).

An ND policy can effectively restrict hazmat shipment from high traffic flow. However, it is also criticized as being rigid since it does not consider the carriers' priorities and wastes the usability of certain road segments. Besides, only restricting certain road segments sometimes cannot rationally adjust the hazmat flows to less-risk areas. Researchers on hazmat transportation has recognized 
the necessity of a more flexible restriction policy of hazmat shipments. Recently, an alternative policy tool, toll-setting policies (TS), was proposed by Marcotte et al. (2009) to deter (but not prevent) hazmat carriers from using certain road segments via toll-pricing. An example given in Marcotte et al. (2009) is helpful to understand that TS policy is a more flexible regulation tool for hazmat than ND policy, and ND policy may be infeasible in certain cases. By imposing tolls on certain road segments, the hazmat shipments are expected to be channeled on less-populated roads according to the carriers' own selection (due to economic considerations) rather than by governors' restriction. This model put forward an attractive policy to network regulators with more flexible solutions, and more acceptable choices to carriers, thereby creating a win-win scenario which is likely to lead to successful implementation.

Toll pricing has been a regular method to control traffic congestion for a long time. When it is infeasible to increase the capacity of the transportation network, imposing appropriate tolls on roads can reduce traffic congestion because tolls can encourage travelers to detour or to travel during a less congested period. Besides, transportation agencies have commonly used tolls to generate revenue to offset infrastructure construction and maintenance cost. Hazmat carriers not only are hazmat traffic flows, but also belong to, and cannot be separated from the regular traffic flows. Therefore, they are managed under more regulations than other kinds of vehicles. That means, the hazmat carriers need to carry pricing on both hazmat and regular tolls. In this sense, this paper proposes a dual-pricing model on both hazmat and regular tolls, trying to channel different kinds of traffic flows to avoid the delays and costs caused by traffic congestion, as well as the probabilities and consequences of the unavoidable vehicle crashes. By separating the hazmat shipments from the heavy-congestion traffic flows, it tries to mitigate the severe accident risks and to avoid peak-time traffic congestions.

The objective of this paper is to develop a dual toll pricing framework to control both regular and hazmat traffic flows for the public safety. The rest of this paper is organized as follows. The next section reviews some popular risk models in hazmat transport and classic congestion models. In Section 3, we present the mathematical formulation of the dual toll pricing model. Section 4 develops an exact solution procedure to solve the dual toll pricing problems and discusses the properties of our model. Section 5 contains our computational experience, based upon data from a real-life hazmat routing situation in the Albany district of New York State. Finally, Section 6 
provides conclusions and future research suggestions.

\section{Literature Review}

In this section, we provide separate literature reviews for congestion pricing methods and hazmat transport routing methods.

\subsection{Literature Review of Congestion Pricing Problems}

A congestion toll pricing problem can usually be classified as first and second best. The first-best policy for a congested road network assumes that tolls equal to marginal external costs on each individual link. In this case, transportation economists often prefer tolls that are based on marginal social cost pricing (Arnott and Small, 1994), with the objective of obtaining a traffic pattern which optimizes the collective use of the network. Hearn and Ramana (1998) define and characterize the set of all tolls which cause drivers to make optimal use of a traffic network, according to specific criteria, e.g., minimize the total amount of tolls collected from the drivers; minimize the number of tolled streets. There are also other first-best tolls that propose models and methodologies for calculating the first best tolls with various (secondary) objective functions, like Dial (1999, 2000), and Hearn and Yildirim (2002) and Yildirim and Hearn (2005). The second-best policy considers the situation that not every single link of a transport network can be tolled. Because such kinds of tolls and other types of restrictions do not generally yield the maximum benefit possible, they are referred to as "second-best" (Johansson-Stenman and Sterner, 1998). These kinds of problems are usually modeled as either a bilevel optimization problem (Bard, 2006), or a mathematical program with equilibrium constraints (MPEC) problem (Luo et al., 1996; Outrata et al., 1998).

Several algorithms have been developed to solve the equivalent nonlinear programming formulations which are equivalent to the MPEC problems. Yan and Lam (1996) present an algorithm for finding optimal tolls for inelastic demands by using derivatives of link flows with respect to tolls only. Hearn and Yildirim (2002) consider the the elastic demands and tolls on subsets of links only, and analyze various additional objectives that can be considered (apart from the maximization of welfare) when there is no unique toll vector decentralizing the system optimum. Fisk and Boyce (1983) offer an alternative variational inequality formulation for an elastic demand traffic 
equilibrium without assuming that each demand function has an inverse. Their analysis concerns only alternative first-best pricing schemes. May and Milne (2000) consider various second-best tolling schemes including cordon, distance-based, time-based and congestion-based charging. They do not, however, consider second-best optimal tolls, but various exogenously determined charge levels instead. Verhoef (2002) proposes a procedure to determine second-best optimal tolls in a generalized network.

In this paper, we assume that every link can be tolled. Our systematical objective is to minimize the overall transportation costs and risk within the whole road network. Our user equilibrium objective is to channel traffic flows along the desired routes generated by the first-best model via toll pricing.

\subsection{Literature Review of Hazmat Transportation}

Route planning for hazmat transportation is usually classified into two general classes: the local route planning problem for each shipment independently, and the global route planning problem for multiple shipments between different O-D pairs on a certain road network. A hazmat carrier is usually concerned with the local routing problem. In this sense, the risk of a hazmat shipment is usually quantified with a path evaluation function.

Much work has been done in the field of hazmat route planning, i.e., planning route choices for hazmat shipments between origin-destination pairs. Usually such kinds of research can be categorized into two fields: local route planning for each shipment independently, and global route planning for multiple shipments between different O-D pairs on a certain road network. Basically two important groups of decision makers are involved in hazardous materials transportation: the governors or network regulators, and the hazmat carriers. The carriers typically focus on the routing of single origin-destination pair (O-D pair) of hazmat shipment. However, a government has to consider the routing problem of all the O-D pair shipments carrying multiple kinds of hazmat types in its jurisdiction. Besides the difference in problem objectives, the two groups of decision makers have different benefit considerations. Usually carriers only consider minimization of the transportation cost, but from the regulator's perspective, the objective is usually the minimization of the social risk brought by hazmat shipments. An overwhelming number of literatures have focused on the assessment of the transportation risk and finding routes to minimize this risk. Many 
risk evaluation models have been developed including minimizing population exposure (ReVelle et al., 1991), expected risk (Alp, 1995), maximal risk (Erkut and Ingolfsson, 2000), probability (Saccomanno and Chan, 1985) or conditional probability (Sivakumar et al., 1993), mean-variance of risk parameters (Erkut and Ingolfsson, 2000), risk disutility (Erkut and Ingolfsson, 2000), or other kinds of risk objectives.

However, because risk mitigation is usually the objective of governors rather than carriers, recently more research focus has moved to balancing the objectives of both governors and carriers to minimize both risks and costs. To address this situation, Kara and Verter (2004) formulated a bilevel ND problem to balance the trade-off between the system optimization objective of minimizing the total risk and the user equilibrium of minimizing each carrier's transportation cost. Building on this work, Erkut and Alp (2007) considered a single-level hazmat transportation ND problem, restricting the network to a tree with only one available path for each origin-destination shipment. Erkut and Gzara (2008) extended this model to the case of an undirected network and proposed a heuristic solution method to overcome the possible lack of stability of the bi-level model solution obtained by solving the single-level mixed integer linear model. All the previous papers adopted a link-based formulation for the carriers' problem, while Verter and Kara (2008) provided a new bi-level path-based formulation for ND problem. A significant issue in developing the path-based model of a hazmat ND problem is the cardinality of alternative path sets, with two extremes corresponding to the most desirable cases for carriers when $\left|P^{s}\right|=1$ for all shipments, and the most ideal scenario for the regulators where all paths between the O-D pairs are included in the model. Although the regulator's ability to mitigate population exposure by closing road segments is not constrained by economic viability, Verter and Kara (2008) propose a way to reach a compromise between the regulator and the carriers to include only paths with lengths that are within a certain percentage of the length of the shortest path.

The literature listed above mainly focuses on ND problems which prohibit the user choice of certain road segments. As we mentioned in Section 1, there are more flexible TS policies to channel hazmat carriers towards lower-density routes via toll-pricing. Although toll pricing has been well developed in traffic congestion problem as we analyzed (see Section 2.1), it is a newly proposed in the hazmat transportation field. Marcotte et al. (2009) put forward a bi-level programming formulation for the hazmat toll-pricing problem via minimizing the risks and costs including both 


\begin{tabular}{ll}
\hline$G(\mathcal{N}, \mathcal{A})$ & a graph \\
$\mathcal{N}$ & set of nodes \\
$\mathcal{A}$ & set of arcs \\
$K$ & set of O-D pairs of regular vehicles \\
$Q$ & set of O-D pairs of hazmat trucks \\
$b$ & demand vector of regular vehicles \\
$d$ & demand vector of hazmat vehicles \\
$v$ & arc flow vector of regular vehicles \\
$u$ & arc flow vector of hazmat trucks \\
$x^{k}$ & arc flow for O-D pair $k \in K$ \\
$y^{q}$ & arc flow for O-D pair $q \in Q$ \\
$s$ & travel cost function \\
$\alpha$ & toll vector for regular vehicles \\
$\beta$ & toll vector for hazmat trucks \\
\hline
\end{tabular}

Table 1: Mathematical notation

transportation costs and tolls forced on hazmats. However, they mainly focus on hazmat tolls to minimize the transportation risks, without considering that hazmat carriers also belong to regular vehicle flows. In this paper, we consider a dual toll pricing system for both hazmat transportation and regular traffic, while limiting and equitably spreading hazmat risk in any zone in which the transportation network is embedded. We examine the feasibility of our models under realistic settings.

\section{Dual Toll Pricing Problem}

Let $G(\mathcal{N}, \mathcal{A})$ be a network with $\mathcal{N}$ being the node set and $\mathcal{A}$ being the arc set. Let $A$ denote the incidence matrix of $G$. Suppose we have both regular and hazmat O-D pair sets, denoted by $K$ and $Q$ respectively. Corresponding to each O-D pair $k \in K$ or $q \in Q$, there is a demand vector $b^{k}$ or $d^{q}$ respectively. For each demand vector $k$ and $q$, there exists arc flow vectors $x^{k}$ and $y^{q}$. The sum of all regular O-D pairs flow vectors is the regular aggregate flow $v$, so is there a hazmat aggregate flow $u$. Table 1 summarizes the notation.

The system defining feasible flows is given by:

$$
V=\left\{v: v=\sum_{k \in K} x^{k}, A x^{k}=b^{k}, x^{k} \geq 0 \quad \forall k \in K\right\}
$$




$$
U=\left\{u: u=\sum_{q \in Q} y^{q}, A y^{q}=d^{q}, y^{q} \geq 0 \quad \forall q \in Q\right\}
$$

Using vector-matrix notations, we rewrite $V$ and $U$ as:

$$
\begin{aligned}
& V=\left\{v: v=Z_{1} x, M_{1} x=b, x \geq 0\right\} \\
& U=\left\{u: u=Z_{2} y, M_{2} y=d, y \geq 0\right\}
\end{aligned}
$$

\subsection{The Model}

Before introducing our model, we need to establish some fundamental assumptions. First, compared to the flow of regular traffics, the number of hazmat trucks is relatively small. Therefore we assume congestion induced by the traffic flow of hazmat trucks can be ignored. Second, to simplify our model, we assume our network users have perfect information of the current status of the network. Third, we only consider a deterministic model, i.e. there is no uncertainty on the travel cost and the behavior of users. Lastly, we only consider one type of hazmat. However, on the basis of the single hazmat problem, an extension to multiple hazmats may be readily implemented.

We are now ready to introduce our model. According to Wardrop's first principle (e.g., Florian and Hearn, 1995), the user, in an attempt to minimizing his or her individual cost, will try the least cost path as he or she sees from a snap-shot of the existing traffic flow. This is called a user equilibrium which can be stated as a variational inequality problem. A traffic flow $\bar{v} \in V$ of regular vehicles is the network user equilibrium if and only if $\bar{v}$ satisfies the following variational inequality:

$$
s(\bar{v}+\bar{u})^{T}(v-\bar{v}) \geq 0 \quad \forall v \in V
$$

where $\bar{u}$ is the current flow of hazmat vehicles. Since we assume no congestion effect due to hazmat trucks, the above variational inequality becomes

$$
s(\bar{v})^{T}(v-\bar{v}) \geq 0 \quad \forall v \in V
$$

Similarly, for a given $\bar{v} \in V$, a traffic flow $\bar{u} \in U$ of hazmat trucks is the network user equilibrium 
when:

$$
s(\bar{v})^{T}(u-\bar{u}) \geq 0 \quad \forall u \in U
$$

The above equilibrium flow $\bar{u}$ is identical to the system optimum flow which is a solution of the following linear program:

$$
\bar{u}=\arg \min _{u \in U}[s(\bar{v})]^{T} u
$$

where the traffic flow of regular vehicles $\bar{v}$ is exogenous.

In the traditional toll pricing model, we try to make users change their behavior by employing toll on roads to achieve the system optimum. After charging a toll $\alpha$, the tolled user optimum problem becomes:

$$
\left(s(\bar{v})^{T}+\alpha\right)(v-\bar{v}) \geq 0 \quad \forall v \in V
$$

Our goal is to make this tolled user optimum as the same as the system optimum. Consider the problem:

$$
\min _{v, u, \alpha, \beta}[s(v)]^{T} v
$$

subject to

$$
\begin{aligned}
(s(v)+\alpha)^{T}(t-v) & \geq 0 \quad \forall t \in V \\
v & \in V \\
\alpha & \geq 0
\end{aligned}
$$

This problem is to be considered to have both $\alpha$ and $v$ as its variables. Then the constraints simply ensure that $v$ is a feasible aggregate flow and it is also a tolled user optimum with respect to toll $\alpha$.

Applying the traditional toll pricing model to our case, we obtain the following problem:

$$
\min _{v, u, \alpha, \beta}[s(v)]^{T} v+[s(v)]^{T} u
$$


subject to

$$
\begin{aligned}
(s(v)+\alpha)^{T}(t-v) & \geq 0 \quad \forall t \in V \\
(s(v)+\beta)^{T}(r-u) & \geq 0 \quad \forall r \in U \\
v & \in V \\
u & \in U \\
\alpha, \beta & \geq 0
\end{aligned}
$$

The above model treats hazmat trucks same as regular traffic; in reality we know that hazmat trucks are much more dangerous than regular traffic. To take this factor into consideration, we introduce one more item risk $R_{a}\left(v_{a}, u_{a}\right)$ for each arc in our formulation besides the travel cost $s(v)$. $R_{a}$ seems to be only caused by hazmat trucks, but the amount of regular traffic on a segment of road would increase the probability of accidents of hazmat trucks. So we write it as a function of both $v_{a}$ and $u_{a}$. Now our objective becomes to minimize

$$
J=w_{1}[R(v, u)]^{T} \mathbb{1}_{A}+w_{2}[s(v)]^{T} v+w_{3}[s(v)]^{T} u
$$

where $w_{1}, w_{2}$ and $w_{3}$ are adjusting parameters for the minimum risk and the minimum system travel cost and $\mathbb{1}_{A}=(1)_{a \in A}$.

In summary our final formulation is:

$$
\min _{v, u, \alpha, \beta} J=w_{1}[R(v, u)]^{T} \mathbb{1}_{A}+w_{2}[s(v)]^{T} v+w_{3}[s(v)]^{T} u
$$

subject to

$$
\begin{aligned}
(s(v)+\alpha)^{T}(t-v) & \geq 0 \quad \forall t \in V \\
(s(v)+\beta)^{T}(r-u) & \geq 0 \quad \forall r \in U \\
v & \in V \\
u & \in U \\
\alpha, \beta & \geq 0
\end{aligned}
$$


This is an instance of Mathematical Programs with Equilibrium Constraints (MPEC), which is in general very difficult to solve. However, due to the structural property, an easy solution method can be devised. In Section 4, we would show the equivalence of this MPEC to a two stage problem which can be much more easily solved.

\subsection{Risk and Cost Functions}

This subsection addresses the definition of risk $R_{a}$ and the property of the objective function under the employment of specific risk function and other parameters. In the rest of this paper, we consider a duration-population-frequency risk function that is similar to the risk function of Cekyay and Verter (2010):

$$
R_{a}\left(v_{a}, u_{a}\right)=s_{a}\left(v_{a}\right) \rho_{a} u_{a}
$$

where $\rho_{a}$ is the population exposure along the arc a.

To simplify the analysis, we consider the following functions, namely:

$$
s_{a}\left(v_{a}\right)=t_{a}\left(1+\left(\frac{v_{a}}{C_{a}}\right)^{\gamma}\right)
$$

The travel delay function (9) is known as the BPR (Bureau of Public Roads) function where $t_{a}$ and $C_{a}$ stand for the free flow time and the capacity of arc $a$ respectively. In this paper, we consider a special case $\gamma=1$.

\section{Reformulation}

In this section, we mainly show that the original problem is equivalent to a two stage problem, and propose methods to solve each problem stage. First, we need the following lemma:

Lemma 1 Let $\bar{v} \in V$, then the following are equivalent:

1. $\bar{v}$ is user optimal, i.e.,

$$
s(\bar{v})^{T}(v-\bar{v}) \geq 0 \quad \forall v \in V
$$


2. there exists $\rho$ such that

$$
\begin{aligned}
& Z^{T} s(\bar{v}) \geq M^{T} \rho \\
& s(\bar{v})^{T} \bar{v}=b^{T} \rho
\end{aligned}
$$

The proof of Lemma 1 is found in Bergendorff et al. (1997).

Now we consider the existence of nonnegative toll vectors that achieve a desired traffic flow pattern as user equilibrium flows. The following lemma is a simple extension of the results of Bai et al. (2006).

Lemma 2 Consider a feasible regular traffic flow pattern $\bar{v} \in V$, and a feasible hazmat traffic flow pattern $\bar{u} \in U$. Then, there exists a pair of nonnegative toll vectors $\alpha \geq 0$ and $\beta \geq 0$ that achives $\bar{v}$ and $\bar{u}$ as tolled user equilibrium flow of regular and hazmat traffic, respectively, if and only if the following hold:

$$
\begin{aligned}
& \bar{v}=\arg \min _{v}\left\{\sum_{a \in \mathcal{A}} \int_{0}^{v_{a}} s_{a}(z) d z: v \in V, v \leq \bar{v}\right\} \\
& \bar{u}=\arg \min _{u}\left\{\sum_{a \in \mathcal{A}} s_{a}\left(\bar{v}_{a}\right) u_{a}: u \in U, u \leq \bar{u}\right\}
\end{aligned}
$$

We further can show that such nonnegative toll vectors always exist when the objective function $J$ is increasing with respect to $v$ and $u$.

Theorem 1 If $J_{a}\left(v_{a}, u_{a}\right)$ is an increasing function of $v_{a}$ and $u_{a}$ for all $a \in \mathcal{A}$, then there exists $a$ pair of nonnegative tolls.

Proof. Suppose $(\bar{v}, \bar{u})=\arg \min _{v \in V, u \in U} J$. Then we can show that $(\bar{v}, \bar{u})$ solves (10) and (11) as similar as in Yin and Lawphongpanich (2006). In particular, suppose $(\hat{v}, \hat{u})$ solves (10) and (11) and $(\bar{v}, \bar{u}) \neq(\hat{v}, \hat{u})$. Because $J_{a}$ is increasing, we obtain

$$
\sum_{a \in \mathcal{A}} J_{a}\left(\hat{v}_{a}, \hat{u}_{a}\right)<\sum_{a \in \mathcal{A}} J_{a}\left(\bar{v}_{a}, \bar{u}_{a}\right)
$$

which is a contradiction because $(\bar{v}, \bar{u})$ minimizes $J$. Therefore, $(\bar{v}, \bar{u})$ solves $(10)$ and $(11)$ and there exists a pair of toll vectors. 
Since our objective function increases (see Section 4.1), we can assure that there always exist toll vectors. Now we can show that our original problem is equivalent to a two-stage problem. For the first-stage problem, we determine the optimal flow to minimize the total risk. That is, we solve the following problem without considering toll vectors:

$$
\min _{v, u} \quad J=w_{1}[R(v, u)]^{T} \mathbb{1}_{A}+w_{2}[s(v)]^{T} v+w_{3}[s(v)]^{T} u
$$

subject to

$$
\begin{aligned}
& v \in V \\
& u \in U
\end{aligned}
$$

In this problem, we do not enforce that the resulting traffic pattern must be from a user equilibrium. Let $\bar{v}$ and $\bar{u}$ denote the solution of this first stage problem.

In the second stage, we determine the toll vectors that make $\bar{v}$ and $\bar{u}$ tolled user equilibrium traffic patterns. Based on Theorem 1 we can see there always exist such a pair of toll vectors $\alpha$ and $\beta$ which can achieve $\bar{v}$ and $\bar{u}$ as tolled user equilibrium.

Therefore our original problem is equivalent to this new two-stage problem. In the following sections, we shall focus on the solution to this two stage problem.

\subsection{The First Stage Problem}

In this subsection, we explore a method to solve the first stage problem. Our objective function is:

$$
\begin{aligned}
J(v, u) & =\sum_{a \in A} J_{a}\left(v_{a}, u_{a}\right) \\
& =\sum_{a \in A}\left\{w_{1} R_{a}\left(v_{a}, u_{a}\right)+w_{2} s_{a}\left(v_{a}\right) v_{a}+w_{3} s_{a}\left(v_{a}\right) u_{a}\right\}
\end{aligned}
$$

With the functions of (8) and (9), $J_{a}$ becomes:

$$
\begin{aligned}
J_{a} & =w_{1}\left(t_{a}+\frac{t_{a}}{C_{a}} v_{a}\right) \rho_{a} u_{a}+w_{2} t_{a}\left(1+\frac{v_{a}}{C_{a}}\right) v_{a}+w_{3} t_{a}\left(1+\frac{v_{a}}{C_{a}}\right) u_{a} \\
& =E_{1}^{a} u_{a}+E_{2}^{a} v_{a}+E_{3}^{a} u_{a} v_{a}+E_{4}^{a}\left(v_{a}\right)^{2}
\end{aligned}
$$


where

$$
\begin{aligned}
& E_{1}^{a}=\left(w_{1} \rho_{a}+w_{3}\right) t_{a} \\
& E_{2}^{a}=w_{2} t_{a} \\
& E_{3}^{a}=\left(w_{1} \rho_{a}+w_{3}\right) \frac{t_{a}}{C_{a}} \\
& E_{4}^{a}=w_{2} \frac{t_{a}}{C_{a}}
\end{aligned}
$$

We can show that the first stage problem is a quadratic programming (QP) problem. The general form for $\mathrm{QP}$ objective function is:

$$
\min \frac{1}{2} x^{T} Q x+c^{T} x
$$

In our case,

$$
\begin{aligned}
v^{T} & =\left[v_{1}, v_{2}, \cdots, v_{n}\right] \\
u^{T} & =\left[u_{1}, u_{2}, \cdots, u_{n}\right] \\
x & =\left[\begin{array}{c}
v \\
u
\end{array}\right] \\
c^{T} & =\left[E_{2}^{1}, E_{2}^{2}, \cdots, E_{2}^{n}, E_{1}^{1}, E_{1}^{2}, \cdots, E_{1}^{n}\right] \\
Q & =\left[\begin{array}{cc}
\operatorname{diag}\left(2 E_{4}^{1}, 2 E_{4}^{2}, \cdots, 2 E_{4}^{n}\right) & \operatorname{diag}\left(E_{3}^{1}, E_{3}^{2}, \cdots, E_{3}^{n}\right) \\
\operatorname{diag}\left(E_{3}^{1}, E_{3}^{2}, \cdots, E_{3}^{n}\right) & 0
\end{array}\right]
\end{aligned}
$$

where $n$ is the number of arcs.

A direct computation shows that half of the eigenvalues of $Q$ are positive and half of them are negative. It is easy to see our objective function is neither convex, concave, nor pseudo-concave. It is obvious that $J$ is not convex. Let us show that $J$ is not pseudoconcave. Schaible (1981) showed that quadratic functions with more than one negative eigenvalue are not quasiconvex or pseudoconvex. Therefore, in our problem, $-J$ cannot be quasiconvex or pseudoconvex.

Although our objective function does not hold a desirable property such as convexity or concavity, there are still several methods available to solve the problem. First, a branch and bound method can be applied to our problem. Falk and Soland (1969) provided one of those for solving 
separable non-convex problems. We employ an orthogonal transformation $x=P y$ where

$$
P^{T} Q P=\operatorname{diag}\left(\lambda_{1}, \lambda_{2}, \cdots, \lambda_{n}\right)
$$

Then our problem can be written in the following form:

$$
\sum_{\lambda_{i}<0} \frac{1}{2} \lambda_{i} y_{i}^{2}+\sum_{\lambda_{i}>0} \frac{1}{2} \lambda_{i} y_{i}^{2}+c^{T} P y
$$

The first term of (14) is a separable concave part and the rest two terms are convex. This makes our objective function fall into the category specified in Falk and Soland (1969):

$$
\min \sum_{i} \varphi_{i}\left(x_{i}\right)+\theta(x)
$$

where $\varphi_{i}$ is separable concave part and $\theta(x)$ is convex. The algorithm suggested in Falk and Soland (1969) relies on the concept of a convex envelope of a non-convex function. For our problem, the convex envelope for the non-convex part on a interval is easy to calculate; it is just the linear function connecting two endpoints. However, Falk and Soland (1969) mentioned that the finite convergence can not be guaranteed if there are convex parts present besides separable concave parts.

The mostly used method for quadratic programming is their null-space active set method. After finding a feasible point during an initial phase, this method searches a solution along the edges and faces of the feasible set by solving a sequence of equality-constrained quadratic programming (ECQP) problems. Next, we give a brief review of how this method works for quadratic programming problem. In the following explanation, we use this formulation of QP :

$$
\min \frac{1}{2} x^{T} Q x+c^{T} x
$$

subject to

$$
\begin{array}{ll}
a_{i}^{T} x=b_{i} & \text { for all } \quad i \in \mathcal{E} \\
a_{i}^{T} x \geq b_{i} & \text { for all } \quad i \in \mathcal{I}
\end{array}
$$


where $Q \in \mathbb{R}^{n \times n}$ is a symmetric matrix and the index sets $\mathcal{I}$ and $\mathcal{E}$ specify the inequality and equality constraints, respectively.

As mentioned above, ECQP problems play an important role in solving general form of quadratic programming. It arises not only as subproblems in solving the general problem, but also in structural analysis and other areas of application. Its formulation is:

$$
\min \frac{1}{2} x^{T} Q x+c^{T} x
$$

subject to

$$
A x=b
$$

Applying the null space method to the above problem,we find a full rank matrix $Z \in \mathbb{R}^{n \times m}$ that spans the null space of $A$. Given a feasible solution $x_{0}$ of ECQP, we can express any other feasible solution as $x=x_{0}+Z w$. Direct calculation leads to an equivalent problem of ECQP:

$$
\min \frac{1}{2} w^{T}\left(Z^{T} Q Z\right) w+\left(Q x_{0}+c\right)^{T} x
$$

If the reduced Hessian matrix $Z^{T} Q Z$ is positive definite, then the solution is same as the following linear equations:

$$
Z^{T} Q Z w=-Z^{T}\left(Q x_{0}+c\right)
$$

Now after the initial phase, given a feasible solution $x_{k}$ to QP, we solve the ECQP subproblem:

$$
\min q\left(x_{k}+d\right)
$$

subject to

$$
a_{i}^{T}\left(x_{k}+d\right)=b_{i} \quad \text { for } \quad i \in \mathcal{W}_{k}
$$


where $q(x)=\frac{1}{2} x^{T} Q x+c^{T} x$, and $\mathcal{W}_{k}$ is the active set of constraints $x_{k}$, i.e

$$
\mathcal{W}_{k}=\left\{i \mid a_{i}^{T} x_{k}=b_{i} i \in \mathcal{E} \cup \mathcal{I}\right\}
$$

If the reduced Hessian matrix of ECQP (15) is positive definite, a solution $x^{*}$ is easily obtained. If $\mathcal{W}_{k}$ is the same as the active constraints at current solution $x^{*}$, a local minimizer is achieved. Otherwise update current feasible solution and $\mathcal{W}_{k}$, continue.

If the reduced Hessian matrix is indefinite, then the subproblem is unbounded below. In this case we need to determine a direction $d_{k}$ such that $q\left(x_{k}+\alpha d_{k}\right)$ is unbounded below, using techniques based on factorizations of the reduced Hessian matrix. Given $d_{k}$, we can update current feasible solution and $\mathcal{W}_{k}$.

\subsection{The Second Stage Problem}

Given the solution of the first stage problem $\bar{v}$ and $\bar{u}$, at the beginning of Section 4, we have shown the existence of a pair of toll vectors $\alpha$ and $\beta$ to achieve $\bar{v}$ and $\bar{u}$ as tolled user equilibrium.

Our goal of charging toll is to change the behavior of network users, meanwhile we want to keep users' cost as low as possible. Therefore among all pairs $(\alpha, \beta)$, we may consider a revenueminimizing toll vector pair, i.e we collect as little toll as possible to minimize financial impacts to the networks. We consider:

$$
\min _{\alpha, \beta, \rho, \omega} \alpha^{T} \bar{v}+\beta^{T} \bar{u}
$$

subject to:

$$
\begin{aligned}
Z_{1}^{T}(s(\bar{v})+\alpha) & \geq M_{1}^{T} \rho \\
(s(\bar{v})+\alpha)^{T} \bar{v} & =b^{T} \rho \\
Z_{2}^{T}(s(\bar{v})+\beta) & \geq M_{2}^{T} \omega \\
(s(\bar{v})+\beta)^{T} \bar{u} & =d^{T} \omega \\
\alpha, \beta & \geq 0
\end{aligned}
$$

The above problem is merely a linear programming problem. We have efficient methods to solve it. 


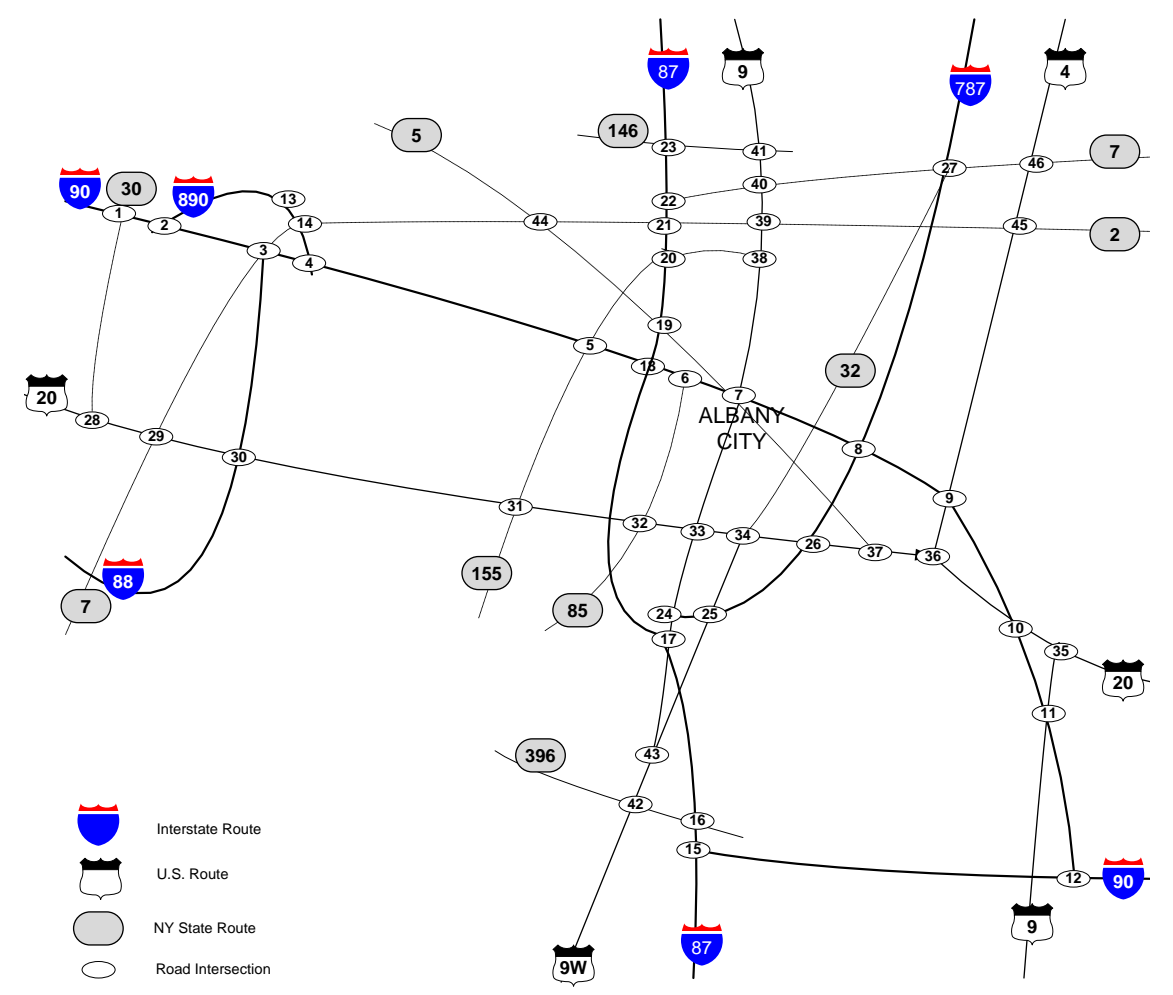

Figure 1: Map of Albany

Moreover, depending on our needs, we can design different kinds of objective function in the above problem. In our numerical example, we consider the above type of objective functions to minimize the total toll.

\section{$5 \quad$ Numerical Example}

In this section, we investigate a part of the road network of Albany in the New York State as shown in Figure 1. The network is comprised of 46 nodes and 70 arcs. We consider two pairs of regular 


\begin{tabular}{|c|c|c|c|c|c|}
\hline$\left(w_{1}, w_{2}, w_{3}\right)$ & $\begin{array}{c}\text { Change of } \\
\text { Risk }\end{array}$ & $\begin{array}{c}\text { Change of } \\
\text { Delay (regular) }\end{array}$ & $\begin{array}{c}\text { Change of } \\
\text { Delay (hazmat) }\end{array}$ & $\begin{array}{c}\text { Tolls Collected } \\
\text { (regular) }\end{array}$ & $\begin{array}{c}\text { Tolls Collected } \\
\text { (hazmat) }\end{array}$ \\
\hline$\left(10^{-4}, 1,1\right)$ & $-15.09 \%$ & $3.95 \%$ & $-0.27 \%$ & $1.29 \times 10^{9}$ & $1.66 \times 10^{3}$ \\
\hline$\left(10^{-3}, 1,1\right)$ & $-22.07 \%$ & $4.68 \%$ & $-1.39 \%$ & $1.31 \times 10^{9}$ & $1.66 \times 10^{3}$ \\
\hline$(1,1,1)$ & $-24.70 \%$ & $22.61 \%$ & $-39.61 \%$ & $5.29 \times 10^{6}$ & 0 \\
\hline$\left(10^{2}, 1,1\right)$ & $-24.70 \%$ & $25.99 \%$ & $-39.52 \%$ & 0 & 0 \\
\hline$\left(10^{5}, 1,1\right)$ & $-24.70 \%$ & $25.99 \%$ & $-39.52 \%$ & 0 & 0 \\
\hline
\end{tabular}

Table 2: Results with various $w_{1}$ with given $w_{2}=1$ and $w_{3}=1$

\begin{tabular}{|c|c|c|c|c|c|}
\hline$\left(w_{1}, w_{2}, w_{3}\right)$ & $\begin{array}{c}\text { Change of } \\
\text { Risk }\end{array}$ & $\begin{array}{c}\text { Change of } \\
\text { Delay (regular) }\end{array}$ & $\begin{array}{c}\text { Change of } \\
\text { Delay (hazmat) }\end{array}$ & $\begin{array}{c}\text { Tolls Collected } \\
\text { (regular) }\end{array}$ & $\begin{array}{c}\text { Tolls Collected } \\
\text { (hazmat) }\end{array}$ \\
\hline$(1,1,1)$ & $-24.70 \%$ & $22.61 \%$ & $-39.61 \%$ & $5.29 \times 10^{6}$ & 0 \\
\hline$\left(1,10^{5}, 1\right)$ & $-5.23 \%$ & $2.86 \%$ & $4.89 \%$ & $2.87 \times 10^{8}$ & 0 \\
\hline$\left(1,10^{8}, 1\right)$ & $-4.41 \%$ & $2.81 \%$ & $5.58 \%$ & $2.62 \times 10^{8}$ & 0 \\
\hline$\left(1,10^{12}, 1\right)$ & $-4.41 \%$ & $2.81 \%$ & $5.58 \%$ & $2.62 \times 10^{8}$ & 0 \\
\hline
\end{tabular}

Table 3: Results with various $w_{2}$ with given $w_{1}=1$ and $w_{3}=1$

demand nodes and three pairs of hazmat demand nodes. The results are obtained by employing a QP solver bqpd which uses null space active set method mentioned in Section 4.1 with a technique for resolving degeneracy (More and Wright, 1993; Fletcher, 1993).

We compare the performance of the dual toll policy with the minimum travel cost flow of regular traffic $\left(v^{s o}\right)$ and the minimum risk flow of hazmat traffic $\left(u^{s o}\right)$ given $v^{s o}$. In particular, we use the

\begin{tabular}{|c|c|c|c|c|c|}
\hline$\left(w_{1}, w_{2}, w_{3}\right)$ & $\begin{array}{c}\text { Change of } \\
\text { Risk }\end{array}$ & $\begin{array}{c}\text { Change of } \\
\text { Delay (regular) }\end{array}$ & $\begin{array}{c}\text { Change of } \\
\text { Delay (hazmat) }\end{array}$ & $\begin{array}{c}\text { Tolls Collected } \\
\text { (regular) }\end{array}$ & $\begin{array}{c}\text { Tolls Collected } \\
\text { (hazmat) }\end{array}$ \\
\hline$\left(10^{-4}, 1,10^{5}\right)$ & $-22.52 \%$ & $3.06 \%$ & $-11.9 \%$ & $3.30 \times 10^{7}$ & 0 \\
\hline
\end{tabular}

Table 4: Results with $\left(w_{1}, w_{2}, w_{3}\right)=\left(10^{-4}, 1,10^{4}\right)$ 
following metric in Tables 2, 3 and 4:

$$
\begin{aligned}
& \text { Change of Risk : } \frac{R\left(u^{*}, v^{*}\right)-R\left(u^{s o}, v^{s o}\right)}{R\left(u^{s o}, v^{s o}\right)} \\
& \text { Change of Delay (regular) }: \frac{s\left(v^{*}\right)^{T} v^{*}-s\left(v^{s o}\right)^{T} v^{s o}}{s\left(v^{s o}\right)^{T} v^{s o}} \\
& \text { Change of Delay (hazmat) }: \frac{s\left(v^{*}\right)^{T} u^{*}-s\left(v^{s o}\right)^{T} u^{s o}}{s\left(v^{s o}\right)^{T} u^{s o}} \\
& \text { Tolls Collected (regular) }: \alpha^{T} v^{*} \\
& \text { Tolls Collected (hazmat) }: \beta^{T} u^{*}
\end{aligned}
$$

where

$$
\begin{aligned}
\left(u^{*}, v^{*}\right) & =\arg \min _{v \in V, u \in U} J \\
v^{s o} & =\arg \min _{v \in V} s(v)^{T} v \\
u^{s o} & =\arg \min _{u \in U}\left\{w_{1}\left[R\left(v^{s o}, u\right)\right]^{T} \mathbb{1}_{A}+w_{2}\left[s\left(v^{s o}\right)\right]^{T} v^{s o}+w_{3}\left[s\left(v^{s o}\right)\right]^{T} u\right\}
\end{aligned}
$$

With these measures, we compare the dual-tolled traffic flows $\left(u^{*}, v^{*}\right)$ with the traffic flows $\left(u^{s o}, v^{s o}\right)$ at regular traffic's system optimum $v^{s o}$.

In the experiments as shown in Table 2 , we vary $w_{1}$ while $w_{2}$ and $w_{3}$ are fixed. We observe that the risk decreases as $w_{1}$ increases, while we have much increased regular traffic delay. With large enough $w_{1}$, we have no tolls collected. Similarly, in Table 3 , we vary $w_{2}$ to put more weight on the delay of regular traffic. With very large $w_{2}$, we reduce the risk while increase the delays. Note that the reduction of risk is achieved by only charging tolls to the regular traffic. In most experiments, the hazmat toll collected is zero. Therefore we can say, in the network considered, that the dual toll pricing problem is about how to charge tolls for regular vehicles rather than how to charge tolls for hazmat trucks.

In Table 4 , we provide a set of weights $\left(w_{1}, w_{2}, w_{3}\right)=\left(10^{-4}, 1,10^{4}\right)$ that significantly reduce the risk while limit the increase of delay. It is achieved by charging only the regular traffic. While this set of weights are determined by trial-and-error, it may be desired to identify a set of Paretooptimal solutions. In such cases, we may consider a genetic algorithm approach that is similar to 
the methods Yin (2002) proposed. Yin and Lawphongpanich (2006) found that genetic algorithm approaches are effective in solving multi-objective toll problems.

\section{Concluding Remarks}

In this paper, we proposed a dual toll pricing model with a linear delay function to reduce the hazmat transportation risk. Our main motivation is to control both regular and hazmat traffic to

reduce the risk. We made a very important assumption: the number of hamzat trucks is relatively small so that the congestion induced by the hazmat trucks can be ignored. We first provided a bi-level formulation and then provided an equivalent two-stage problem. While the second-stage problem is an easily solved linear programming problem, the first-stage problem is a non-convex quadratic problem. We suggested two methods to solve the first stage problem of our model: the branch and bound, and the null space active set method. As a numerical example, we studied the road network of the Albany, NY, U.S.A. We observed that our dual toll can reduce the hazmat transportation risk significantly, while it limits the increase of transportation costs to a reasonable level.

Although we only considered the linear travel delay function in this paper, our dual toll pricing framework can be extended to problems with more general delay functions, such as the BPR function with order of 2 or 4 . However, for such cases, the first-stage problem will become highly non-linear and non-convex. A heuristic approach may be developed to solve such problems.

\section{References}

Alp, E. (1995). Risk-based transportation planning practice: Overall methodology and a case example. Information Systems and Operational Research 33(1), 4-19.

Arnott, R. and K. Small (1994). The economics of traffic congestion. American Scientist 20(2), $123-127$.

Bai, L., D. W. Hearn, and S. Lawphongpanich (2006). Relaxed toll sets for congestion pricing problems. In S. Lawphongpanich, D. W. Hearn, and M. J. Smith (Eds.), Mathematical and Computational Models for Congestion Charging. Springer. 
Bard, J. F. (2006). Practical Bilevel Optimization: Algorithms and Applications (Nonconvex Optimization and Its Applications). Secaucus, NJ, USA: Springer-Verlag New York, Inc.

Bergendorff, P., D. Hearn, and M. Ramana (1997). congestion toll pricing of traffic newworks. Network Optimization, 51-71.

Cekyay, B. and V. Verter (2010). Transportation of hazardous materials in urban areas. Working Paper, McGill University.

Dial, R. B. (1999). Minimal-revenue congestion pricing part i: A fast algorithm for the single-origin case. Transportation Research Part B: Methodological 33(3), 189-202.

Dial, R. B. (2000). Minimal-revenue congestion pricing part ii: An efficient algorithm for the general case. Transportation Research Part B: Methodological 34(8), 645-665.

Erkut, E. and O. Alp (2007). Designing a road network for hazardous materials shipments. Computers \& Operations Research 34(5), 1389-1405.

Erkut, E. and F. Gzara (2008). Solving the hazmat transport network design problem. Computers E Operations Research 35(7), 2234-2247.

Erkut, E. and A. Ingolfsson (2000). Catastrophe avoidance models for hazardous materials route planning. Transportation Science 34(2), 165.

Falk, J. E. and R. M. Soland (1969, May). An algorithm for separable nonconvex programming problems. Management Science 15(9), 550-569.

Fisk, C. S. and D. E. Boyce (1983). Alternative Variational Inequality Formulations of the Network Equilibrium-Travel Choice Problem. Transportation Science 17(4), 454-463.

Fletcher, R. (1993). Resolving degeneracy in quadratic programming. Annals of Operations Research 46(2), 307-334.

Florian, M. and D. Hearn (1995). Network equilibrium models and algorithms. Handbooks in Operations Research and Management Science 8, 485-550. 
Gallamore, R., C. Barkan, B. Bowditch, Jr., J. Brinson, M. Bronzini, O. Bugg, C. Burke, E. Chapman, T. Egan, N.J.Garber, P. Kelley, M. Moreland, M. Morrissette, G. Veerman, and T.R. Menzies, Jr. (2005). Cooperative Research for Hazardous Materials Transportation: Defining the Need, Converging on Solutions. Special Report 283, Transportation Research Board.

Hearn, D. and M. Ramana (1998). Solving congestion toll pricing models. In P. Marcotte and S. Nguyen (Eds.), Equilibrium and advanced transportation modeling, pp. 109-124. Kluwer Academic Publishers.

Hearn, D. W. and M. B. Yildirim (2002). A Toll Pricing Framework for Traffic Assignment Problems with Elastic Demand, pp. 135-145. Kluwer Academic Publishers.

Johansson-Stenman, O. and T. Sterner (1998). What Is the Scope for Environmental Road Pricing?., pp. 150 - 170. Gothenburg U: Cheltenham, U.K. and Northampton, Mass.

Kara, B. and V. Verter (2004). Designing a road network for hazardous materials transportation. Transportation Science 38(2), 188-196.

Luo, Z.-Q., J.-S. Pang, and D. Ralph (1996). Mathematical programs with equilibrium constraints. Cambridge University Press, Cambridge ; New York :.

Marcotte, P., A. Mercier, G. Savard, and V. Verter (2009). Toll Policies for Mitigating Hazardous Materials Transport Risk. Transportation Science 43(2), 228-243.

May, A. D. and D. S. Milne (2000, August). Effects of alternative road pricing systems on network performance. Transportation Research Part A: Policy and Practice 34(6), 407-436.

More, J. and S. Wright (1993). Optimization software guide. Society for Industrial Mathematics.

Movassaghi, K., J. Daly, T. Dinh-Zarr, S. Herbel, P. Joavanis, L. Porter, H. Robertson, T. Songer, P. Sweatman, R. Tay, T. Welch, and T. Menzies, Jr. (2007). Building the road safety profession in the public sector. Special Report 289, Transportation Research Board.

Outrata, J., M. Kocvara, and J. Zowe (1998). Nonsmooth Approach to Optimization Problems with Equilibrium Constraints. Kluwer Academic Publishers,Dordrecht, The Netherlands. 
ReVelle, C., J. Cohon, and D. Shobrys (1991). Simultaneous siting and routing in the disposal of hazardous wastes. Transportation Science 25(2), 138-145.

Saccomanno, F. and A. Chan (1985). Economic evaluation of routing strategies for hazardous road shipments. Transportation Research Record 1020, 12-18.

Schaible, S. (1981, November). Quasiconvex, pseudoconvex, and strictly pseudoconvex quadratic functions. Journal of Optimization Theory and Applications 35(3), 303-338.

Sivakumar, R., R. Batta, and M. Karwan (1993). Establishing credible risk criteria for transporting extremely dangerous hazardous materials. In F. Saccomanno and K. Cassidy (Eds.), Transportation of Dangerous Goods: Assessing the Risks, pp. 335-342. Institute for Risk Research, University of Waterloo, Canada.

Verhoef, E. (2002). Second-best congestion pricing in general static transportation networks with elastic demands. Regional Science and Urban Economics 32(3), 281-310.

Verter, V. and B. Y. Kara (2008). A Path-Based Approach for Hazmat Transport Network Design. Management Science 54(1), 29-40.

Yan, H. and W. H. K. Lam (1996). Optimal road tolls under conditions of queueing and congestion. Transportation Research Part A: Policy and Practice 30(5), 319-332.

Yildirim, M. B. and D. W. Hearn (2005). A first best toll pricing framework for variable demand traffic assignment problems. Transportation Research Part B: Methodological 39(8), 659 - 678.

Yin, Y. (2002). Multiobjective bilevel optimization for transportation planning and management problems. Journal of Advanced Transportation 36(1), 93-105.

Yin, Y. and S. Lawphongpanich (2006). Internalizing emission externality on road networks. Transportation Research Part D: Transport and Environment 11(4), 292-301. 